\title{
Development of Neural Sensitivity to Face Identity Correlates with Perceptual Discriminability
}

\author{
Vaidehi S. Natu, ${ }^{1}$ Michael A. Barnett, ${ }^{1}$ Jake Hartley, ${ }^{1}{ }^{-}$Jesse Gomez, ${ }^{2}{ }^{\circledR}$ Anthony Stigliani, ${ }^{1}$ and Kalanit Grill-Spector ${ }^{1,2,3}$ \\ ${ }^{1}$ Department of Psychology, ${ }^{2}$ Neurosciences Program, and ${ }^{3}$ Stanford Neurosciences Institute, Stanford University, Stanford, California 94305
}

Face perception is subserved by a series of face-selective regions in the human ventral stream, which undergo prolonged development from childhood to adulthood. However, it is unknown how neural development of these regions relates to the development of faceperception abilities. Here, we used functional magnetic resonance imaging (fMRI) to measure brain responses of ventral occipitotemporal regions in children (ages, 5-12 years) and adults (ages, 19-34 years) when they viewed faces that parametrically varied in dissimilarity. Since similar faces generate lower responses than dissimilar faces due to fMRI adaptation, this design objectively evaluates neural sensitivity to face identity across development. Additionally, a subset of subjects participated in a behavioral experiment to assess perceptual discriminability of face identity. Our data reveal three main findings: (1) neural sensitivity to face identity increases with age in face-selective but not object-selective regions; (2) the amplitude of responses to faces increases with age in both face-selective and object-selective regions; and (3) perceptual discriminability of face identity is correlated with the neural sensitivity to face identity of face-selective regions. In contrast, perceptual discriminability is not correlated with the amplitude of response in face-selective regions or of responses of object-selective regions. These data suggest that developmental increases in neural sensitivity to face identity in faceselective regions improve perceptual discriminability of faces. Our findings significantly advance the understanding of the neural mechanisms of development of face perception and open new avenues for using fMRI adaptation to study the neural development of high-level visual and cognitive functions more broadly.

Key words: development; face morphs; face perception; fMRI adaptation; high-level visual cortex

\section{Significance Statement}

Face perception, which is critical for daily social interactions, develops from childhood to adulthood. However, it is unknown what developmental changes in the brain lead to improved performance. Using fMRI in children and adults, we find that from childhood to adulthood, neural sensitivity to changes in face identity increases in face-selective regions. Critically, subjects' perceptual discriminability among faces is linked to neural sensitivity: participants with higher neural sensitivity in face-selective regions demonstrate higher perceptual discriminability. Thus, our results suggest that developmental increases in face-selective regions' sensitivity to face identity improve perceptual discrimination of faces. These findings significantly advance understanding of the neural mechanisms underlying the development of face perception and have important implications for assessing both typical and atypical development.

\section{Introduction}

Face perception is subserved by a series of face-selective regions in the ventral stream, including a region in the inferior occipital

Received June 7, 2016; revised Aug. 18, 2016; accepted Sept. 1, 2016.

Author contributions: V.S.N. and K.G.-S. designed research; V.S.N., M.A.B., J.H., J.G., A.S., and K.G.-S. performed research; V.S.N., A.S., and K.G.-S. analyzed data; V.S.N. and K.G.-S. wrote the paper.

This work was supported by National Institutes of Health Grants 1R01EY 02231801A1 and 1R01EY02391501A1 to K.G.-S., and 5T32EY020485 to V.S.N., and by National Science Foundation Graduate Research Fellowship Program Grant DGE-114747 to J.G.

The authors declare no competing financial interests.

Correspondence should be addressed to Vaidehi S. Natu, Department of Psychology, Stanford University, Stanford, CA 94305. E-mail: vnatu@stanford.edu.

DOI:10.1523/JNEUROSCI.1886-16.2016

Copyright $($ C) 2016 the authors $\quad 0270-6474 / 16 / 3610893-15 \$ 15.00 / 0$ gyrus (Gauthier et al., 2000) and two regions on the lateral fusiform gyrus (Kanwisher et al., 1997; McCarthy et al., 1997; GrillSpector et al., 2004; Weiner and Grill-Spector, 2010; Parvizi et al., 2012). Fusiform face-selective regions undergo prolonged development from childhood to adulthood (Aylward et al., 2005; Golarai et al., 2007, 2010, 2015; Scherf et al., 2007; Peelen et al., 2009; Cantlon et al., 2011) associated with age-related increases in (1) the spatial extent of face-selective regions, (2) the response amplitude to faces, and (3) the degree of selectivity to faces versus nonfaces. Children also demonstrate a protracted development of face-perception ability (Carey et al., 1980; de Heering et al., 2012; Weigelt et al., 2014). However, it is unknown what brain changes underlie this perceptual development. Here, we fill this 
a
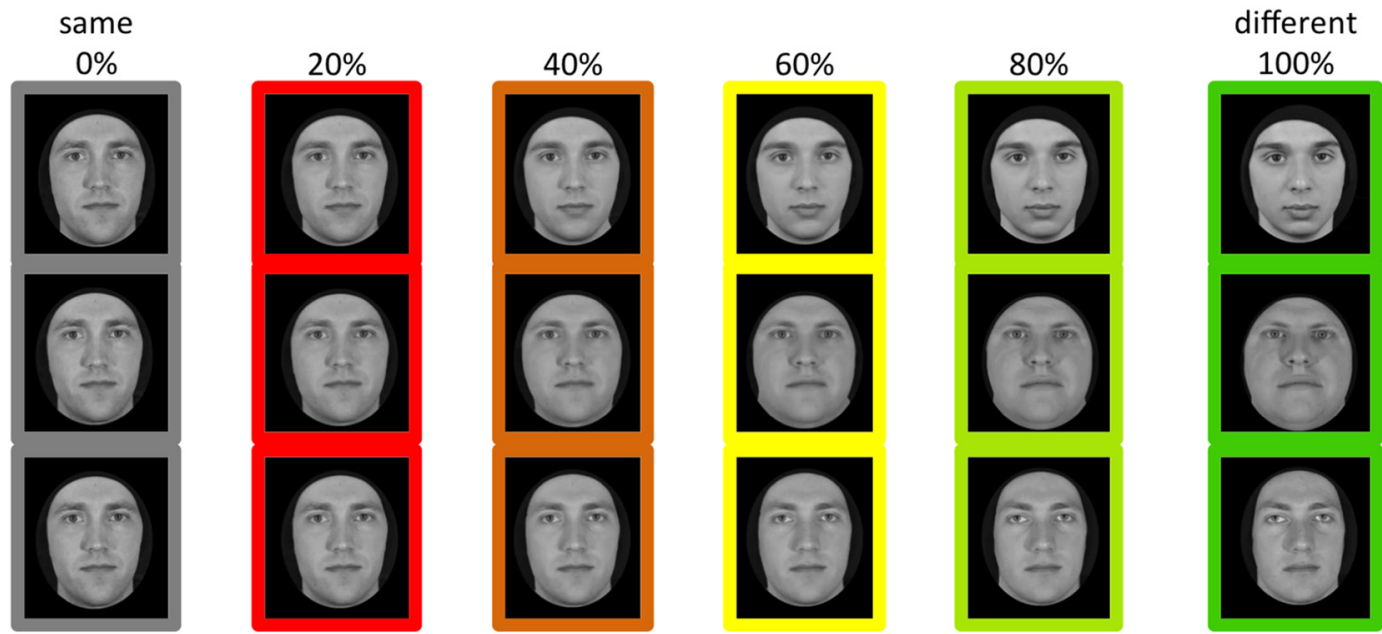

b

$100 \%$ different female child faces

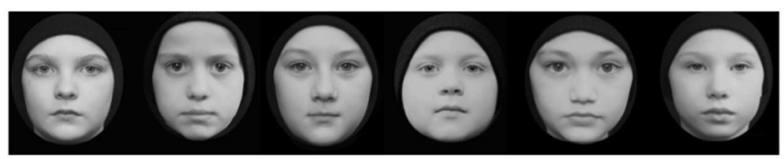

$100 \%$ different female adult faces

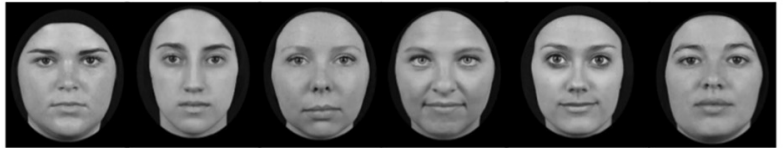

C

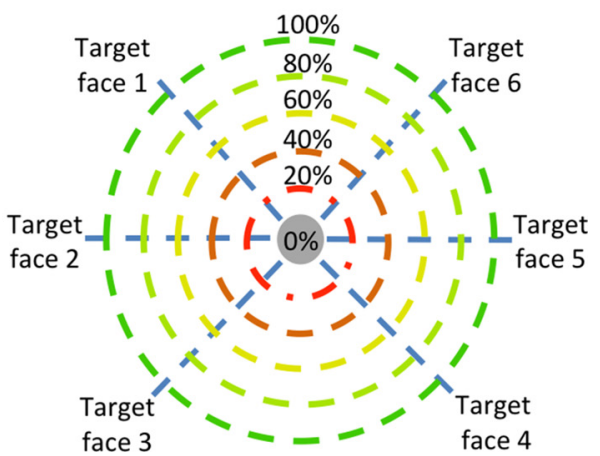

Figure 1. Experimental design. $\boldsymbol{a}$, Example stimuli used in the study. Each column shows sample stimuli from an adult male block at a single morph level. During the experiment, six stimuli were shown in random order in a block. Note that in $0 \%$ morph the same face is shown and, as morph level increases, the faces in a block become more dissimilar. Rows illustrate how identity varies from the source to target face. $\boldsymbol{b}$, Example stimuli show a block of $100 \%$ different female child faces in the top row and a block of $100 \%$ different female adult faces in the bottom row. $\boldsymbol{c}$, Schematic of the face-morph space. Each source face was morphed into six target faces from the same gender and age along a straight morph line, with a linear weighting of the source and target faces. Each circle denotes stimuli shown in a block at each morph level. Morph level indicates percentage weighting of the target face.

gap in knowledge by examining the development of neural mechanisms of perceptual discrimination of faces.

In adults, perceptual judgments of face identity are correlated with the sensitivity of face-selective regions to changes in face identity (Rotshtein et al., 2005; Jiang et al., 2006; Gilaie-Dotan and Malach, 2007; Gilaie-Dotan et al., 2010). Functional magnetic resonance imaging (fMRI) adaptation (fMRI-A) reveals reduced responses in ventral face-selective regions to identical faces than to different faces (Grill-Spector et al., 1999; Grill-Spector and Malach, 2001; Avidan et al., 2002; Winston et al., 2004; Ewbank and Andrews, 2008). Notably, the level of fMRI-A depends on the similarity among faces. That is, more-similar faces, compared with less-similar faces, yield more fMRI-A (Jiang et al., 2006; Davidenko et al., 2012). Therefore, from these measurements we can derive the level of change in brain responses as a function of stimulus similarity, which we refer to as neural sensitivity. Based on findings in adults, we hypothesized that throughout development, the neural sensitivity of face-selective regions to facial identity increases and consequently perceptual discrimination of faces improves. This hypothesis predicts (1) higher neural sensitivity to face identity in adults' face-selective regions than in children's face-selective regions and (2) a correlation between neural sensitivity and perceptual discriminability to face identity.
A second possibility is that the developmental improvement in face perception is associated with stronger responses to faces. Stronger responses in adults' face-selective regions compared with those in children may provide a higher neural signal-tonoise ratio regime, which in turn may facilitate better perceptual judgment (Reynolds and Heeger, 2009). Indeed, responses to faces in adults' fusiform face-selective regions are higher than those in children's fusiform face-selective regions, and in both age groups there is an own-age bias, whereby responses are higher for own-age than other-age faces (Golarai et al., 2015). This neural own-age bias may be associated with the behavioral own-age bias in face recognition (Harrison and Hole, 2009; Hills and Lewis, 2011). Thus, an alternative hypothesis is that development is not associated with changes in neural sensitivity but rather increases in the level of responses to faces in face-selective regions, which then improves perceptual discrimination. Of course, a third possibility is that improved perceptual discrimination involves developmental increases in both neural sensitivity and responses to faces.

To test these hypotheses, we used fMRI in 23 children (5-12 year olds) and 12 adults (19-34 year olds), to measure responses in face-selective regions when subjects viewed child and adult faces that parametrically varied in their similarity (Fig. 1). From these measurements we (1) derived neural sensitivity to face 
identity and baseline response amplitudes to faces, and (2) tested whether there are age-related differences in each of these metrics. To evaluate whether neural development has perceptual consequences, we obtained perceptual similarity ratings in a subset of children and adults, and tested whether perceptual discriminability is related to neural sensitivity and/or response amplitude to faces. Finally, to test whether these developments are specific to face-selective regions or are broader, we repeated these measurements in two object-selective regions. One region, LO, was located in the lateral occipital cortex, posterior to hMT + . The other region, $\mathrm{pFs}$, was located on the posterior fusiform and the occipital temporal sulcus (Malach et al., 1995; Grill-Spector et al., 1999).

\section{Materials and Methods Subjects}

We collected neuroimaging data from 23 children (ages, 5-12 years; 14 females) and 12 adults (ages, 19-34 years; 5 females). Children were recruited from the Palo Alto school district through flyers and on-line advertisements in school newsletters. All children attended public schools at the time of recruitment. Adult subjects are university affiliates and do not engage with children as part of their daily work. Only 1 of 12 adult participants is a parent and is living with their 5-year-old child. MR images were collected using a 3T Signa scanner (GE Healthcare) in the Stanford Center for Cognitive and Neurobiological Imaging (CNI) at Stanford University. All subjects had normal or corrected-to-normal vision and provided written, informed consent. Protocols were approved by the Stanford Internal Review Board on Human Subjects Research.

\section{Data collection sequence}

All subjects underwent several sessions, which included an anatomy scan, two functional neuroimaging scans (for the localizer and face morph experiments), and a behavioral testing session outside the scanner. Additionally, all children underwent a separate training session, before the scanning sessions, inside a mock MRI scanner. In the mock scanner, children were trained to remain still by monitoring their motion, using a motion sensor attached to their forehead and providing them with feedback of the amount of head movement they made. Children who stayed still during the mock session were invited back for MRI scans.

\section{MRI acquisition}

\section{Anatomical scans}

Neuroimaging data were acquired from a 3T Signa scanner (GE Healthcare) at CNI using a custom-built phase-array 32-channel receive-only head coil. To obtain whole-brain, high-resolution anatomical scans, we used the methods described in a previous study (Mezer et al., 2013) to produce T1-weighted anatomies from quantitative scans. Specifically, quantitative T1 parameters were measured from spoiled gradient echo images acquired with different flip angles $\left(\alpha=4,10,20\right.$, and $30^{\circ} ; \mathrm{TR}=20$ $\mathrm{ms} ; \mathrm{TE}=2.4 \mathrm{~ms}$ ) and a voxel resolution of $0.8 \times 0.8 \times 1 \mathrm{~mm}$, which was resampled to $1 \mathrm{~mm}^{3}$ isotropic. For T1 calibration, subjects were scanned using spin-echo inversion recovery with an echo-planar imaging (EPI), read-out (SEIR-EPI). This scan was done with a slab-inversion pulse and spatial-spectral fat suppression. For SEIR-EPI, the TR was $3 \mathrm{~s}$. The echo time was set to minimum full; inversion times were 50,400, 1200, and $2400 \mathrm{~ms}$. We used $2 \mathrm{~mm}^{2}$ in-plane resolution with a slice thickness of 4 $\mathrm{mm}$ and the EPI readout was performed using $2 \times$ acceleration. Anatomical data were aligned to the anterior commissure-posterior commissure plane.

\section{Functional scans}

Functional scans were obtained with the same scanner and coil using a $\mathrm{T} 2{ }^{\star}$-sensitive gradient echo spiral pulse sequence with a resolution of $2.4 \times 2.4 \times 2.4 \mathrm{~mm}$; TR $=1000 \mathrm{~ms}$; TE $=78.6 \mathrm{~ms}$; flip angle, $76^{\circ}$; and FOV, $192 \mathrm{~mm}$. We collected 48 oblique slices, oriented parallel to the superior temporal sulcus, using a multiplexing technique enabling whole-brain coverage of functional data (Feinberg et al., 2010). The same prescription was used to obtain whole-brain anatomical T1-weighted images (in-plane scan), which were used to align functional data with the high-resolution anatomical volume of each participant. During MRI scanning, participants lay supine inside the magnet. Visual stimuli were projected onto a monitor and were viewed through an angled mirror mounted above the participant's head.

\section{Stimuli}

Stimuli for the face-morph and behavioral experiments consisted of human faces of male and female children and adults. A subset of the children's faces was obtained from the Dartmouth Database of Children's Faces (Dalrymple et al., 2013) and the remaining child faces and all of the adult faces were photographed in our laboratory. Photography sessions were conducted in an enclosed room under controlled lighting to match the Dartmouth database. Specifically, participants wore a black cape covering their clothing and a black beanie covering their hair. Participants did not have prominent facial hair (e.g., beard) or glasses. Using a highresolution digital single-lens reflex D90 Nikon camera, frontal views of people's faces were photographed against a wall covered with black felt. Forty-two child faces ( 21 female faces) and 42 adult faces ( 21 females faces) were photographed. The average age of the child faces was $9 \pm 1.7$ years and that of the adult faces was $23 \pm 3.1$ years. All images were converted to gray scale, preprocessed to remove any remaining hair, moles, or spots using Adobe Photoshop CS4, and matched for luminance using the Matlab-based SHINE (spectrum, histogram, and intensity normalization and equalization) toolbox (Willenbockel et al., 2010).

Each morph set consisted of seven face identities, whereby one face (source face) was morphed with six other faces (target faces) from the same age group and gender. We created six morph face sets for each of the child and adult faces. Half of the morph sets consisted of female faces and half of males. Face morphs were generated using the Morph Age 4.1.3 toolbox. Two faces (source and target faces) were morphed together by aligning features, including the eyes, eyebrows, nose, nostrils, lips, and the external contour. For each source-target face pair, the Morph Age tool produced a QuickTime movie file with 120 frames, showing the source face transforming into the target face. We selected six frames per source-target morph line, starting from frame 1, followed by every 20th frame thereafter, thereby obtaining six morph levels for each morph pairing: $0 \%$ morph, a face that contains $100 \%$ of the source face and $0 \%$ of the target face; $20 \%$ morph, a face that contains $80 \%$ of the source face and $20 \%$ of the target face; and $40,60,80$, and $100 \%$ morph levels in the same manner.

Images from morph lines with the same source face were grouped together into blocks of six faces (Fig. 1c, each concentric circle) for both fMRI and behavioral experiments. Each block contained images of a single morph level. Thus, the $0 \%$ block contained six presentations of the source face (Fig. 1a, 0\% column, sample triplets), the $20 \%$ block contained six different faces ( $80 \%$ a single source, $20 \%$ different targets; Fig. $1 a, 20 \%$ column), and the $100 \%$ block contained six different individuals (Fig. 1a, last column, $b$, sample $100 \%$ different female child and adult faces). Consequently, with increasing morph level the variability of faces in a block increases (Fig. $1 c$, radii of the circles). For example, faces in the $20 \%$ morph level are highly similar to each other; however, those in the $60 \%$ morph level appear dissimilar (Fig. 1a, 60\% column for sample triplets). We generated phase-scrambled images using the original and morphed adult and child face stimuli to serve as targets for an oddball task during fMRI.

\section{fMRI experiments}

\section{Functional localizer}

This experiment was used to define face-selective regions based on higher responses to faces than nonfaces. Face-selective regions of interest (ROIs) were defined in individual subjects using a localizer experiment based on methods reported in our prior publications (Stigliani et al., 2015). All subjects participated in three runs of an independent functional localizer experiment $(5.24 \mathrm{~min} / \mathrm{run})$ with $78,4 \mathrm{~s}$ blocks in each run. During the experiment, subjects viewed gray-scale stimuli, which were blocked by category. Images consisted of two subtypes from each of five categories: 
characters (numbers and pseudowords), bodies (limbs and headless bodies), human faces (child faces and adult faces), places (houses and indoor scenes), and objects (guitars and cars). Each image was shown once and the faces used in the localizer were different from those used in the face-morph experiment. In each $4 \mathrm{~s}$ block, different stimuli of one of the above categories were shown at a rate of two images per second. Blocks were counterbalanced across categories and also with baseline blocks consisting of a blank, gray background. In the oddball task, subjects fixated on a central dot and pressed a button when a phasescrambled oddball image appeared randomly in a block ( $\sim 33 \%$ of the blocks). Due to equipment malfunction, we were able to collect behavioral data during scan only on six children (ages, 7-12 years; six females) and five adults (ages, 24-34 years; two females). Children's accuracy at the oddball task was $81.0 \pm 10.5 \%$, which was not significantly different from the adults' accuracy at this task (84.4 $\pm 9.65 \%)$.

\section{Lateral occipital complex localizer}

This experiment was used to define object-selective regions that respond more strongly to intact than to scrambled objects (Malach et al., 1995; Grill-Spector et al., 1998, 1999). On a session conducted on a different day, 10 of 12 adults and 16 of 23 children participated in an independent lateral occipital complex (LOC) localizer experiment. The experiment contained 10 blocks of abstract 3D objects that alternated with blocks of scrambled objects. Each $12 \mathrm{~s}$ block contained images presented at a rate of $1 \mathrm{~Hz}$ and lasted $4.4 \mathrm{~min}$. In the one-back task, subjects were asked to press a button if the same image repeated twice in a row (33\% of the blocks).

\section{Face-morph experiment}

The goal of the face-morph study was to examine (1) whether neural sensitivity to changes in face identity varies as a function of the age of subject and (2) whether neural sensitivity to changes in face identity differs for own-age faces versus other-age faces. We used an fMRI-A paradigm (Grill-Spector et al., 1999) in which blocks varied in the level of dissimilarity among faces (Fig. $1 a$, columns). We hypothesized that fMRI-A would be largest for the $0 \%$ condition, in which subjects viewed six identical stimuli in a block, and lowest for the $100 \%$ condition, in which subjects viewed six different faces (consequently this block should have the highest responses), with intermediate fMRI-A for intermediate morph levels.

During fMRI, participants viewed stimuli in two runs (4 min each). Each run contained 18 blocks of child faces, 18 blocks of adult faces, and 18 fixation blocks. Blocks were $4 \mathrm{~s}$ long and consisted of six faces of a single morph level presented at a rate of two images per second, followed by $1 \mathrm{~s}$ of fixation. Each run began and ended with $12 \mathrm{~s}$ of fixation. Each of the six morph levels occurred three times during a run, for each of the child and adult face blocks, and contained a different morph set. The block order was counterbalanced such that consecutive blocks never displayed stimuli from the same morph set or morph level. Additionally, the appearance of images from the same morph set were pseudorandomized such that the next appearance of the same morph set would be in a block that was different by $\geq 2$ morph steps from the preceding one (e.g., $0-40 \%, 100-20 \%)$.

\section{Oddball task}

Subjects fixated on a central dot and pressed a button when a phasescrambled oddball image appeared randomly in a block ( $\sim 33 \%$ of the blocks). Due to equipment malfunction we were able to collect behavioral data during scan only on eight children ages 5-12 years (five females). Children's accuracy at the oddball task was $89.25 \pm 4.7 \%$ (mean $\pm \mathrm{SE}$ ), demonstrating that children attended the stimuli and were proficient at performing the task.

\section{Behavioral testing of perceptual discriminability}

Eleven of 12 adult participants and 12 of 23 children underwent behavioral testing outside the scanner on a different day (average gap between fMRI and behavioral testing: $28.2 \pm 15.3 \mathrm{~d}$ ). This experiment was conducted to examine (1) whether perceptual discriminability varies across children and adults, (2) whether perceptual discriminability is higher for own-age faces than for other-age faces, and (3) whether there is a rela- tionship between perceptual discriminability and neural sensitivity to changes in face identity as measured in the face-morph study.

During the behavioral experiment, participants viewed trials consisting of six simultaneously presented faces from a single morph level (Fig. $1 b$ ). Subjects rated the similarity of these six faces on a scale of 1 to 5 , where " 1 " indicated "exactly same," and " 5 " indicated "different." Trials were self-paced. Each participant viewed 120 trials, 20 per morph level, which were chosen randomly from 216 possible trials shown in the fMRI experiment. All subjects saw both adult and child faces of both genders spanning all morph levels.

\section{Data analysis}

\section{Analysis of anatomical data}

Quality assurance. We evaluated the quality of the anatomical images. Subjects whose anatomical data appeared blurry or showed ringing due to motion were asked to return for a second scan on a different day $(N=3$; mean age, 6.7 years). The second scan was of sufficient quality and was used for subsequent visualization and cortical surface reconstruction.

Reconstruction of cortical surfaces. The T1 anatomical images of each participant were segmented into white and gray matter using the FreeSurfer automatic segmentation tool (http://surfer.nmr.mgh.harvard. edu). White matter surfaces were inspected and manually fixed for missing or mislabeled white matter voxels using ITK-SNAP (http://www. itksnap.org/). A mesh of each participant's cortical surface was generated from the boundary of the white and gray matter. This mesh was inflated for visualization of activations inside the sulci.

\section{Analysis of functional data}

The localizer and face-morph data were analyzed using code written in Matlab-based mrVista toolbox (http://github.com/vistalab) as in our prior publications (Weiner and Grill-Spector, 2010, 2011; Golarai et al., 2015; Stigliani et al., 2015). Data were not spatially smoothed and were analyzed in each subject's native brain space. The time courses of each voxel were converted from arbitrary scanner units into units of percentage signal change.

Motion correction. Data of each subject were corrected for within-run and between-run motion. In both the localizer and face-morph experiments, only datasets with motion of $<2$ voxels were included in the study (as in our prior studies: Golarai et al., 2007, 2010, 2015). Four children were excluded from further analysis because of excessive motion during fMRI scans. Despite this strict motion criterion, we observed that children have larger motion inside the scanner than adults $\left(F_{(1,59)}=11.99\right.$, $p<0.01$, children moved on an average $0.74 \pm 0.64$ voxels during scan compared with adults, who moved only $0.33 \pm 0.28$ voxels during scan). Therefore, we conducted a more conservative analysis by comparing fMRI data from the face-morph experiment on 12 adults and 12 of 19 children matched for their motion (no significant difference among groups: $\left.F_{(1,45)}=3.41 ; p>0.05\right)$. This matching was accomplished by removing seven children with the highest motion under two voxels. Results remained consistent with those reported when considering all subjects' data.

General linear model. For each subject's data, we ran a general linear model (GLM) to model each voxel's time course. The experimental design matrix was convolved with the SPM hemodynamic response function (http://www.fil.ion.ucl.ac.uk/spm) to generate predictors. Using a GLM to fit the predictors to the data, we estimated the response amplitudes for each condition $(\beta)$ and residual variance of each voxel's time course. We used the $\beta$ values and residual variance from the GLM to generate contrast maps comparing responses in different conditions.

\section{Analysis of localizer data}

Face-selective voxels were defined as voxels that responded more to faces (adult and child) than images of other categories $(t>3$, voxel level) during the localizer scan. We defined in each subject three face-selective regions in the ventral stream as in our prior studies (Weiner and GrillSpector, 2010, 2011; Stigliani et al., 2015): (1) a region in the inferior occipital gyrus (IOG faces), also referred to as the occipital face area (Gauthier et al., 2000); (2) a region in the posterior fusiform gyrus (pFus 
a

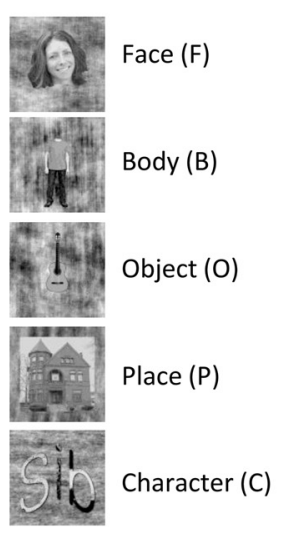

b

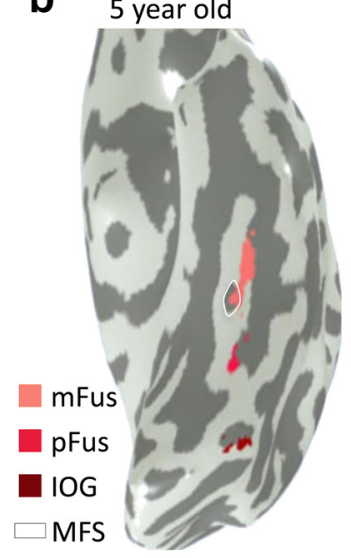

12 year old

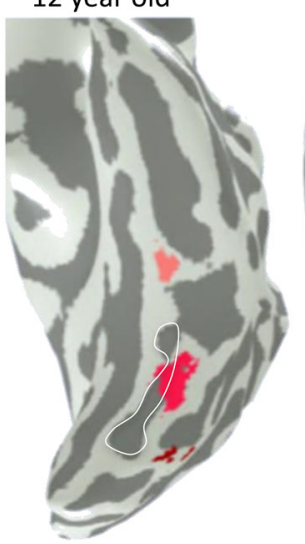

24 year old

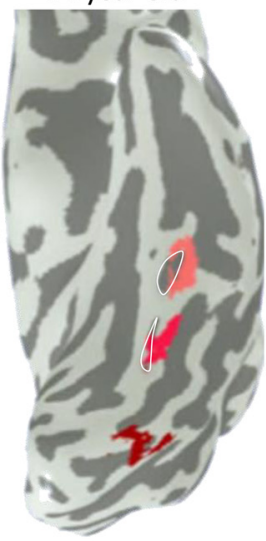

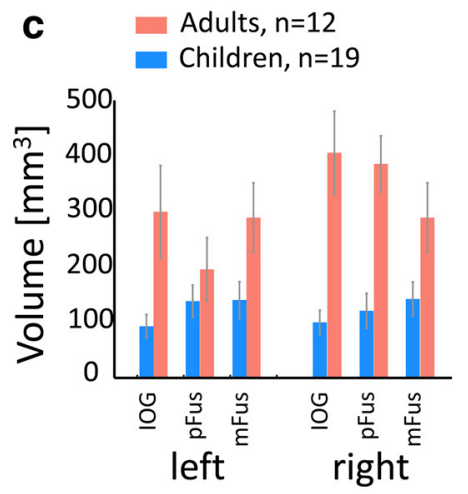

d

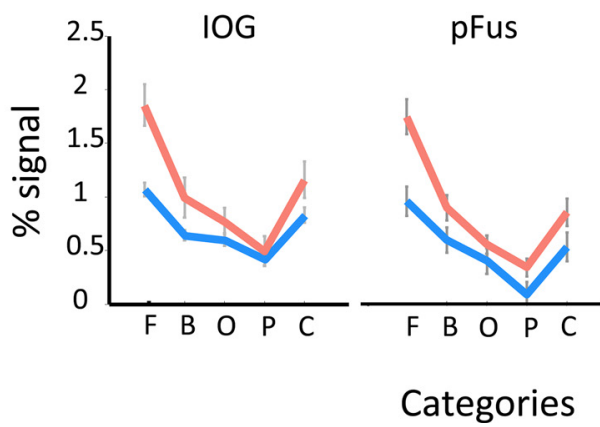

mFus

Figure 2. Development of face-selective regions in the ventral stream. $\boldsymbol{a}$, Example face, body, object, place, and character stimuli used in the localizer experiment. $\boldsymbol{b}$, Face-selective regions in the ventral occipitotemporal cortex in the left hemisphere of three example subjects. Face-selective regions were defined using the contrast faces $>$ objects, places, words, bodies ( $t>3$, voxel level), in the inferior occipital gyrus (IOG faces, dark red), in the posterior fusiform (pFus faces, red), and mid-fusiform (mFus faces, pink). White contour, mid fusiform sulcus (MFS). $c$, The average volume of face-selective regions (IOG, pFus, and mFus) in left and right hemispheres of 19 children (blue) and 12 adults (orange). Adult face-selective regions are significantly larger than those of children $\left(F_{(1,157)}=62.91, p<0.001\right)$. Error bars, standard error of mean (SEM) across participants of an age group. $\boldsymbol{d}$, Independent analysis of percentage signal change in the three face-selective regions across five stimulus categories depicted in $\boldsymbol{a}$. Result reveals a significant age-by-category interaction $\left(F_{(4,410)}=8.43, p<0.001\right)$ and significantly higher responses for faces in adults than in children $\left(F_{(1,82)}=55.61, p<0.001\right)$. Error bars, SEM across participants of an age group. $F$, Face; $B$, body; 0 , object; $P$, place; $C$, character.

faces), also referred to as FFA-1; and (3) a region in the mid-fusiform gyrus (mFus faces) also referred to as FFA-2. The combined ROI of FFA-1 and FFA-2 is referred to as the fusiform face area (FFA; Kanwisher et al., 1997).

Independent analysis of response amplitudes

In each subject, we conducted an independent analysis of the response amplitudes during the localizer by applying a GLM to two of three runs of the localizer to define the face-selective ROIs using the contrast faces $>$ bodies, objects, places, and characters $(t>3$, voxel level). Then we extracted the response amplitude (percentage signal change) per category from the left-out run. We repeated this analysis across three iterations of choosing two of three runs and averaged the responses per category, across iterations. Results of this analysis are shown in Figure $2 d$.

To test for developmental changes in the volume of ROIs as reported previously (Golarai et al., 2007, 2015; Scherf et al., 2007), we measured the volume of each face-selective region in each subject and compared across age groups (Fig. 2c).

\section{Analysis of LOC localizer}

We defined in each subject two object-selective regions that responded more strongly to intact objects than scrambled objects ( $t>3$, voxel level). One region, LO, was located in the lateral occipital cortex, posterior to $\mathrm{hMT}+$, and one region, $\mathrm{pFs}$, was located ventrally on the posterior fusiform and the occipital temporal sulcus. Since the contrast of objects $>$ scrambled is not mutually exclusive from the contrast faces $>$ nonfaces, we excluded object-selective voxels that overlapped face-selective voxels.
Data from 2 of 16 children were not used for further analysis, due to excessive motion during this experiment.

\section{Analysis of face-morph data}

Response amplitudes for experimental conditions were derived from $\beta$ s estimated from the GLM analysis.

Evaluating $f M R I$ responses as a function of dissimilarity among face identities in children and adults. The goal of this analysis was to examine whether the sensitivity of the neural responses in face-selective regions to the level of dissimilarity among faces (using the entire range of six morph levels from 0 to $100 \%$ ) varied across age groups (adult/child participants) and/or age of face (adult/child face). For each subject and ROI, we measured the amplitude of fMRI responses as a function of morph level. We fit a linear model to each subject's responses separately for the stimuli depicting child faces and adult faces. From each subject's linear fit, we evaluated the slope, which we refer to as the neural sensitivity to changes in face identity, and the intercept, which we refer to as the baseline response for identical faces (i.e., neural response when the same face is presented on the screen). Thus, a slope and an intercept were estimated for each participant per stimulus type (adult or child face) and per ROI (IOG, pFus, and mFus faces). To test whether developmental changes were unique to face-selective regions or occurred more broadly across high-level visual areas, the same analyses were repeated for object-selective $\mathrm{LO}$ and $\mathrm{pFs}$.

Slopes and intercepts were used as dependent measures in a three-way repeated-measures ANOVA, with age of subject, age of face, and ROI as 
independent measures. We repeated the three-way ANOVA on the slopes and intercepts for the subset of 12 children and 12 adults matched for motion (see above, Motion correction).

Evaluating whether low-level differences between face types contribute to $f M R I$ measurements. In this analysis, we tested whether physical imagebased dissimilarities between the child and adult faces affected neural sensitivity to faces. Although we carefully matched the low-level image differences by matching luminance across images, we measured imagebased differences between face types using the structural similarity algorithm (Wang et al., 2004), available in the SHINE toolbox (Willenbockel et al., 2010). This algorithm considers differences in luminosity, contrast, and structure between the pair of images and generates a structural similarity index (SSIM) ranging from 0 to 1 for each image pair (where SSIM $=1$ for two identical images, and SSIM $=0$ for maximally different images). We then converted the SSIM to an image dissimilarity index (IDI; IDI $=1-$ SSIM) and calculated the average IDI across all image pairs in each morph level block. Child faces were more dissimilar than adult faces at morph levels ranging between 20 and $100 \%\left(F_{(1,60)}=55.68\right.$, $p<0.001)$. For example, the IDI for $100 \%$ different child faces was 0.20 which differed from that for $100 \%$ different adult faces that was $0.14\left(t_{(10)}\right.$ $=2.9, p<0.05)$ and the IDI of $60 \%$ morph of child faces was matched to the IDI of $100 \%$ morph of adult faces $\left(t_{(10)}=0.8, p>0.05\right)$. To test whether IDI contributes to responses in face-selective regions, we computed the level of fMRI-A, measured as the difference between the response amplitude to condition of interest and the response amplitude to $0 \%$ morph showing repetitions of the same identity, for IDI-matched stimuli (60\% morphs of child faces and $100 \%$ different adult faces) and for the $100 \%$ child faces and $100 \%$ adult faces. We reasoned that if IDI contributes to $\mathrm{AMRI}-\mathrm{A}$, then the level of $\mathrm{AMRI}-\mathrm{A}$ in face-selective regions should be identical for IDI-matched adult and child faces, but there will be a larger release from adaptation for blocks containing $100 \%$ child faces compared with $100 \%$ adult faces, as the former condition has higher IDI. In these analyses, fMRI-A was measured in each subject and ROI separately for blocks containing child and adult faces. We conducted a threeway repeated-measures ANOVA across all face-selective ROIs (IOG, pFus, and mFus faces) and tested whether fMRI-A varied as a function of the age of subject (child/adult), age of face (child face/adult face), or both.

Time series signal-to-noise ratio analysis. As a control to test whether developmental changes were driven by nonspecific differences across age groups, we evaluated whether there were differences in time series signalto-noise ratio (tSNR) across children and adults. For each subject, we measured the tSNR during the face-morph experiment in each of the face-selective regions: IOG, pFus, and mFus faces. tSNR was computed for each voxel as follows: $\mathrm{tSNR}=$ mean(time series)/SD(time series) and then averaged across all voxels in each ROI per subject, and across subjects in an age group.

\section{Analysis of perceptual discriminability of faces}

Data from the behavioral experiment conducted outside the scanner was analyzed as follows: Each subject provided a dissimilarity score (ranging from 1 to 5 , " 1 " being exactly same, and " 5 " being different) for 20 trials from each morph level. For each subject, we calculated the average dissimilarity score for each morph level separately for the child and adult faces. We conducted a three-way ANOVA, with age of subject (child/ adult), age of face (child face/adult face), and morph level as factors to test for developmental effects. We also tested whether there are differences in the distribution of response ratings across children and adults. Results show that both adults and children report " $1=$ same" responses more often than the other responses (main effect of rating, $F_{(4,105)}=$ $24.21, p<0.001$ ), but there are no significant differences in the distribution of ratings across age groups.

To test whether there are significant differences across age groups in perceptual discriminability, we fit each subject's perceptual dissimilarity ratings separately for child and adult faces using linear fits and then compared whether the slopes of the linear fits significantly differ across age groups and face types using a two-way ANOVA with factors of subject age group and age of face stimulus. The slope (which we refer to as perceptual discriminability) indicates the behavioral sensitivity to detect identity changes-larger slopes indicate higher sensitivity. Data are shown in Figure 8. We also fit the behavioral data with a Weibull function (Zychaluk and Foster, 2009) replicating the results of the linear fits (data not shown).

\section{Evaluating the relationship between perceptual discriminability and neural sensitivity}

To relate behavioral data to the neural response amplitudes, we calculated the correlation between perceptual sensitivity (slope of perceptual dissimilarity ratings) and neural sensitivity (slope of fMRI data during the face morph experiment). Since the neural data were fitted with a linear fit, and a linear fit provides a reasonable fit for the behavioral data, we compared perceptual and neural slopes estimated from the linear fit. We also evaluated the relationship between perceptual discriminability and response amplitudes to faces. We report data for the three faceselective ROIs-IOG, pFus, and mFus faces_-for child and adult faces separately.

Additionally, we ran a control analysis to test whether the relationship between perceptual discriminability and neural sensitivity is driven by outlier subjects that have negative neural sensitivity to face identity. First, we identified subjects who have negative mean neural sensitivity in both pFus faces and mFus faces for both face types. This analysis identified three children that have consistently negative slopes across these ROIs and face types. We then repeated the correlation analysis mentioned above without these subjects.

\section{Results}

\section{The spatial extent of face-selective regions develops from childhood to adulthood}

In both children and adults, we localized three face-selective regions in the ventral occipitotemporal cortex (Fig. 2b). One region was on the inferior occipital gyrus (IOG faces, right and left hemisphere combined, $\left.N_{\text {child }}=17 / 19, N_{\text {adult }}=12 / 12\right)$, one in the posterior fusiform gyrus (pFus faces, right and left hemisphere combined, $N_{\text {child }}=17 / 19, N_{\text {adult }}=12 / 12$ ), and one in the mid fusiform gyrus ( $\mathrm{mFus}$ faces, right and left hemisphere combined, $N_{\text {child }}=18 / 19, N_{\text {adult }}=12 / 12$ ). The volume of face-selective regions was larger in adults than in children (Fig. $2 c$, main effect of age: $\left.F_{(1,157)}=62.91, p<0.001\right)$. There were no significant differences across hemispheres or ROIs.

Independent analysis of response amplitudes in face-selective regions during the localizer experiment revealed higher response amplitudes to faces as well as higher selectivity to faces in adults than children. As expected, responses in face-selective regions of both children and adults varied across categories, and were highest for faces (Fig. $2 d$; main effect of category: $F_{(4,410)}=93.04, p<$ 0.001; three-way ANOVA with factors of age group, category, and ROI). Additionally, response amplitudes to faces in face-selective regions were higher in adults than in children, and differences across age groups were larger for faces than for other categories (age of subject by category interaction, $F_{(4,410)}=8.43, p<0.001$ ).

Results of the localizer experiment show the following: (1) we are able to localize face-selective regions in each child and adult; (2) the spatial extent of face-selective regions is larger in adults than in children, replicating prior results (Aylward et al., 2005; Golarai et al., 2007; Scherf et al., 2007); and (3) there is a larger development of response amplitudes to face stimuli than to other stimuli in face-selective regions, which is consistent with prior findings (Golarai et al., 2007, 2010, 2015; Scherf et al., 2007).

\section{Are there differences across age groups in the neural sensitivity to faces?}

To test whether there are developmental changes (1) in neural sensitivity to variations in face identity, or (2) in the amplitude of response to faces independent of face variability, or (3) in both, we conducted the face-morph experiment in which we measured 


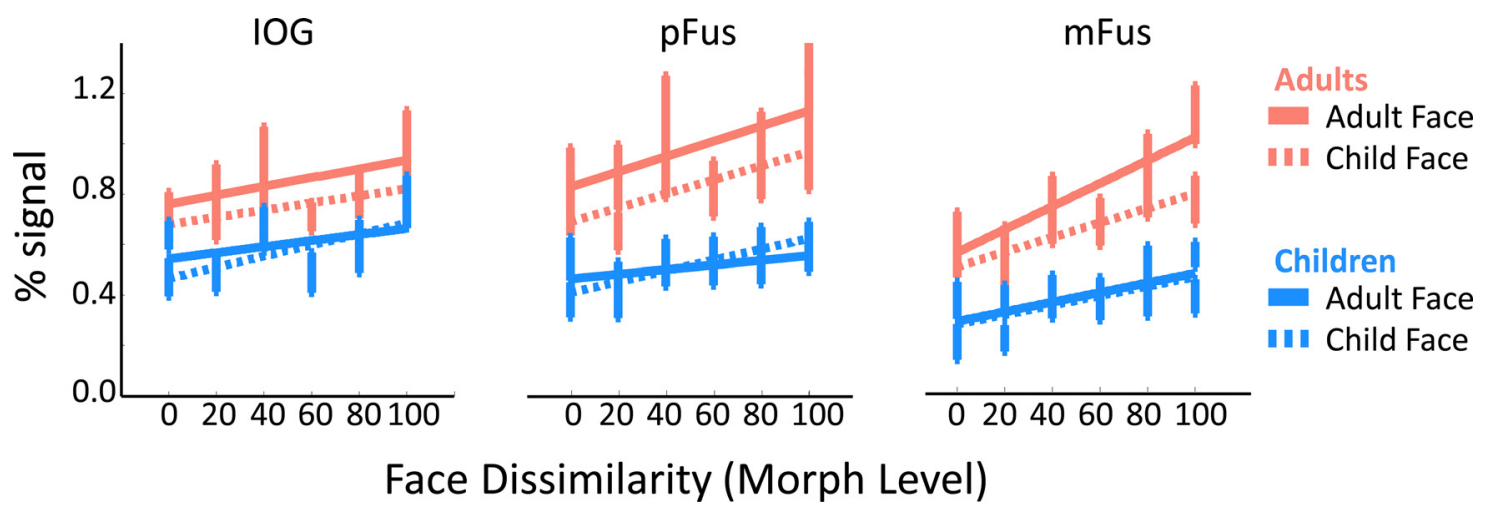

Figure 3. Responses in face-selective regions as a function of face dissimilarity (morph level) during the face-morph study. Mean percentage signal in face-selective regions in adults (orange; IOG, $n=12 ; \mathrm{pFus}, n=12 ; \mathrm{mFus}, n=12$ ) and children (blue; I0G, $n=17 ; \mathrm{pFus}, n=17 ; \mathrm{mFus}, n=18$ ) as a function of morph level. Solid lines, Responses to adult faces; dashed lines, responses to child faces. Error bars, SEM.

responses of face-selective regions in adults and children (defined individually using the localizer) as a function of dissimilarity among faces (Fig. 1).

In both children and adults, responses in ventral face-selective regions systematically increased with increasing face dissimilarity (Fig. 3). That is, in both age groups, responses to blocks in which identical faces were presented were the lowest, responses to blocks in which $100 \%$ different faces were presented were the highest, and there was a systematic increase in the response amplitude of face-selective regions with increasing dissimilarity among faces in a block [significant main effect of morph level: $F_{(5,984)}=17.89, p<0.01$; four-way ANOVA on percentage signal change with factors of morph level $(0-100 \%)$, age of subject (child/adult), age of face (child face/adult face), and ROI (IOG/ pFus/mFus) ]. Additionally, across all face-selective ROIs, morph levels, and face types, responses to faces in children were significantly lower than responses in adults (main effect of age of subject: $\left.F_{(1,984)}=267.28, p<0.01\right)$. Notably, across ROIs and face types the highest responses were observed in adults' face-selective regions when viewing blocks of 100\% different adult faces, and the lowest responses where observed in children's face-selective regions when viewing blocks of identical faces ( $0 \%$ morph).

To quantitatively evaluate developmental changes, we fit a linear model to each subject's responses in each face-selective region. From the linear fit, we evaluated the slope of the linear fit, which indicates neural sensitivity to face identity (Fig. $4 a$ ), and intercept, which indicates the baseline response amplitude for faces (Fig. 4b). Then we compared these measurements across age groups.

Comparison of slopes from different age groups reveals three findings. First, we found that in both children and adults the slopes of IOG, pFus, and mFus faces were significantly positive ( $t s>5$, ps $<0.05)$. This shows that blocks with more dissimilar faces produce higher signals than blocks with similar faces and there is a systematic relationship between face dissimilarity and neural responses. Second, comparison of slopes across age groups revealed significantly larger slopes in face-selective regions of adults than of children (Fig. $4 a$; main effect of age: $F_{(1,164)}=5.4$, $p=0.02$; three-way ANOVA with factors of age of subject, age of face, and ROI). This indicates that adults' face-selective regions are more sensitive to face dissimilarity than those of children. Third, there were no significant differences in slopes to own-age faces than other-age faces (interaction between age of subject and age of face: $\left.F_{(1,164)}=2.75 ; p=0.099\right)$. Sensitivity to face identity was similar when comparing all three face-selective regions: IOG,
pFus, and mFus faces (no significant two-way or three-way interactions between ROI and age of subject or age of face). However, neural sensitivity to face identity was significantly larger in mFus faces than IOG faces [main effect of ROI: $F_{(1,110)}=4.75 ; p=0.03$; three-way ANOVA on slopes with factors age of subject, age of face, and ROI (IOG/mFus)].

Comparison of intercepts of the linear fits in IOG, pFus, and mFus faces across age groups revealed that adults, compared with children, have significantly higher intercepts (Fig. 4b, main effect of age: $F_{(1,164)}=51.3, p<0.001$; three-way ANOVA with factors age of subject, age of face, and ROI). There were no significant differences in intercepts for own-age versus other-age faces (no significant age of subject-by-age of face interaction: $F_{(1,164)}=$ $0.37, p>0.05)$, and there were no other significant two-way or three-way interactions $(F s<0.8, p s>0.05)$. This shows that the baseline response to faces in face-selective regions of adults is higher than those of children regardless of the age of face.

Since the ages of the child participants spanned a range between 5 and 12 years, it is possible that they are not a homogeneous group and that there are qualitative differences in neural responses between younger and older children in this age range. Thus, we generated scatterplots of each of the parameters of neural responses (slope/intercept) versus child age. Results indicated no qualitative differences between younger and older children in either slopes or intercepts (Fig. 5). Further, there was no significant correlation of either slope or intercept with age in either children's IOG or pFus faces, for either type of face (correlation slope with age: IOG, $-0.2<R<-0.15$, ps $>0.38$; pFus, $-0.4<$ $R<-0.2$; ps $>0.11$; correlation intercept with age: IOG, $0.09<$ $R<0.21$, ps $>0.41$; pFus, $0.33<R<0.39$, ps $>0.11$; Fig. 5). However, in mFus faces we found a significant negative correlation between the slope of neural sensitivity with age when children viewed adult faces $(R=-0.71, p<0.001)$, whereby older children had lower neural sensitivity than younger ones. In contrast, we found no significant correlation when children viewed child faces (correlation of slope with age: $R=0.22, p>0.36$ ). Additionally, intercepts of baseline response to faces in mFus faces significantly increased with age when children viewed both child and adult faces $(R s>0.61 ; p s<0.007)$. Finally, in our adults (ages, 19-34 years) there was no significant correlation with age for either slope or intercept in any of the ROIs and for both face types (slopes: $0.15<R<0.44$, ps $>0.15$; intercepts: $-0.1<R<0.3$, ps $>0.25$ ) except for a significant increase in the baseline response to child faces in mFus faces $(R=0.72, p<$ 0.007 ). Results of these analyses showing no qualitative differ- 

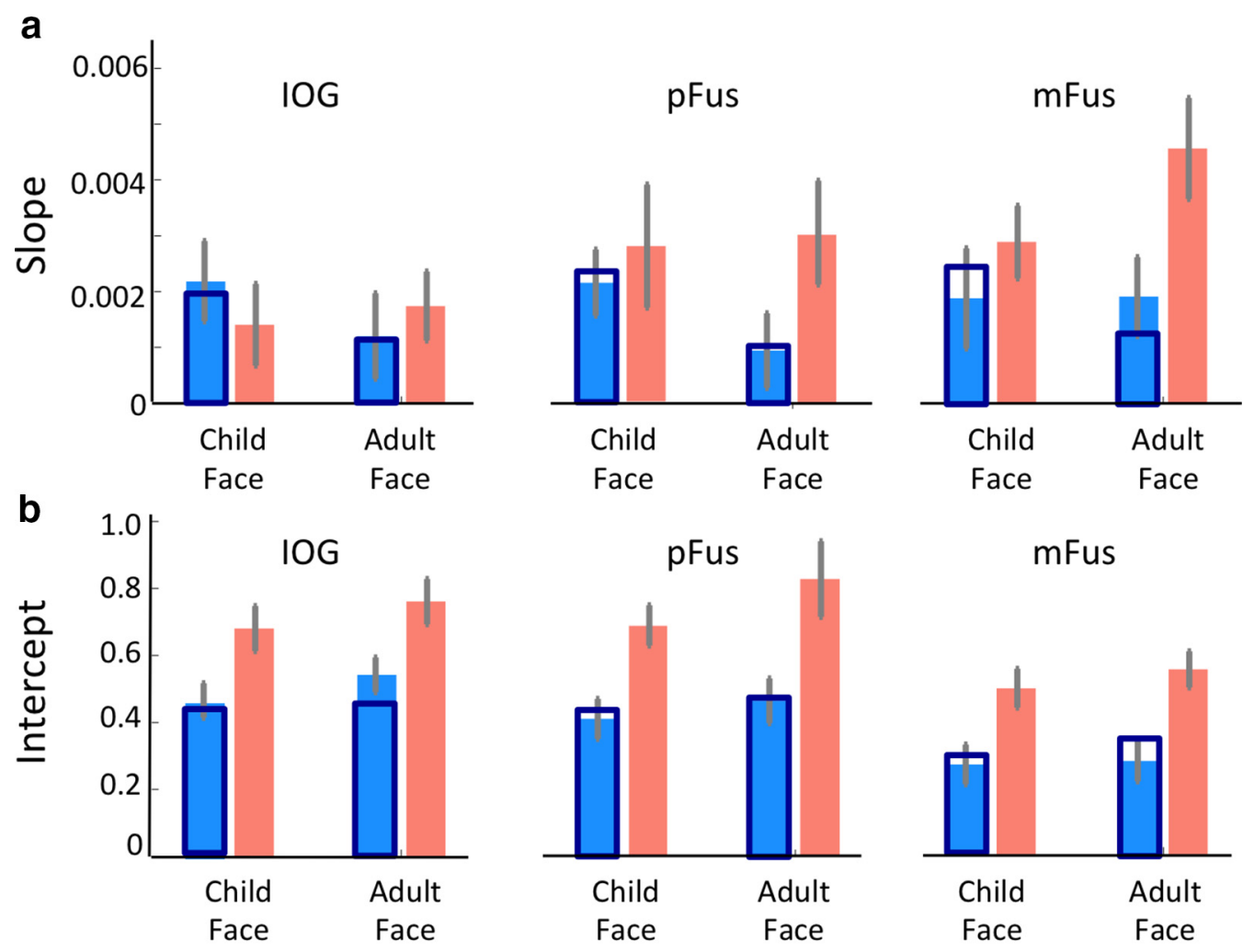

Children

Motion-matched children

Adults

Figure 4. Slopes and intercepts of linear fits to response amplitudes in face-selective regions during the face-morph study. $\boldsymbol{a}$, Slopes, indicating the neural sensitivity to face identity, were estimated in each subject and then averaged across participants of an age group, separately for each ROl and face stimulus. Slopes were significantly higher in adults than in children $\left(F_{(1,164)}=5.4\right.$, $p=0.021)$. Orange, Adults; light blue, children; dark blue, subset of children motion-matched to adults. Results remained the same for motion matched subjects $\left(F_{(1,132)}=5.44, p<0.05\right)$. There was also a significant interaction between age of subject and age of face $\left(F_{(1,132)}=4.37, p<0.05\right)$ in the motion-matched data. Error bars, SEM averaged across participants of an age group (adults: IOG, $n=12 ; \mathrm{pFus}, n=12 ; \mathrm{mFus}, n=12$; children: IOG, $n=17 ; \mathrm{pFus}, n=17 ; \mathrm{mFus}, n=18) \cdot \boldsymbol{b}$, Intercepts, indicating the baseline response level for child and adult faces in each of the age groups, were estimated in each subject, and then averaged across participants of an age group, separately for each ROl and face stimulus. Intercepts are significantly larger in adults than in children $\left(F_{(1,164)}=51.3, p<0.001\right)$. Orange, Adults; light blue, children; dark blue, subset of children motion-matched to adults; results remained the same $\left(F_{(1,132)}=42.83, p<0.001\right)$. Error bars, SEM averaged across participants within an age group.
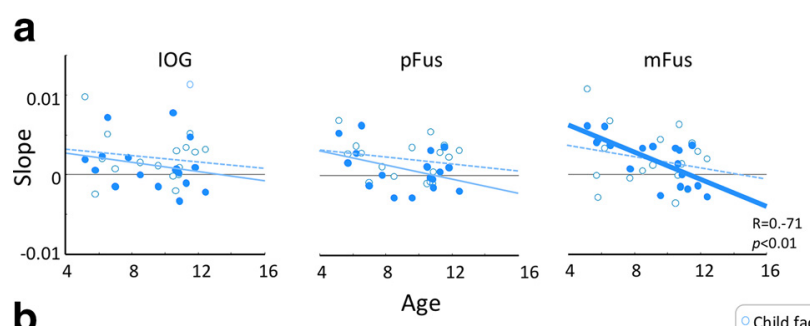

b
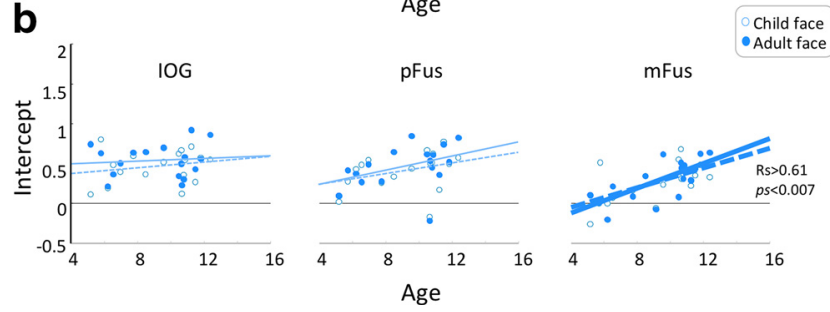

Figure 5. Scatterplot of slopes and intercepts of linear fits to response amplitudes in faceselective regions versus age of child participants. $\boldsymbol{a}$, Slopes, indicating neural sensitivity to face identity for child and adult faces as a function of child age for IOG, pFus, and mFus faces. Each point is a participant. $\boldsymbol{b}$, Intercepts, indicating the baseline response level for child and adult faces as a function of child age for IOG, pFus, and mFus faces. Each point is a participant. Open circles, Child faces; filled circles, adult faces; dashed lines, regression analysis for child faces; solid lines, regression analysis for adult faces; bold lines, significant correlations; statistics are indicated in the right. ences between younger and older children, and progressive changes with age restricted to mFus faces, suggest that it is largely valid to compare the group of children in these age ranges to the group of adults.

\section{Are the observed differences between age groups driven by higher noise in children?}

To test whether age-related differences in slopes or intercepts are due to noisier measurements in children than adults, we first tested whether there are differences across age groups in tSNR or motion during fMRI, and then repeated analyses on a subset of subjects matched on these noise metrics (Grill-Spector et al., 2008). The mean tSNR in children's face-selective regions (IOG, pFus, and mFus) was $56.15 \pm 14.5$, which was not significantly different $\left(F_{(1,82)}=2.88, p>0.05\right)$ than the mean tSNR in adults $(52.24 \pm 12.78)$.

While all subjects included in the prior analyses moved $<2$ voxels during scan, which was our motion threshold for inclusion, children moved on an average $0.74 \pm 0.64$ voxels during scan compared with adults, who moved only $0.33 \pm 0.28$ voxels during scanning (main effect of age: $F_{(1,59)}=11.99, p<0.01$ ). Greater motion in children may cause partial voluming between face-selective regions and adjacent regions that are not faceselective, consequently reducing the observed level of response 


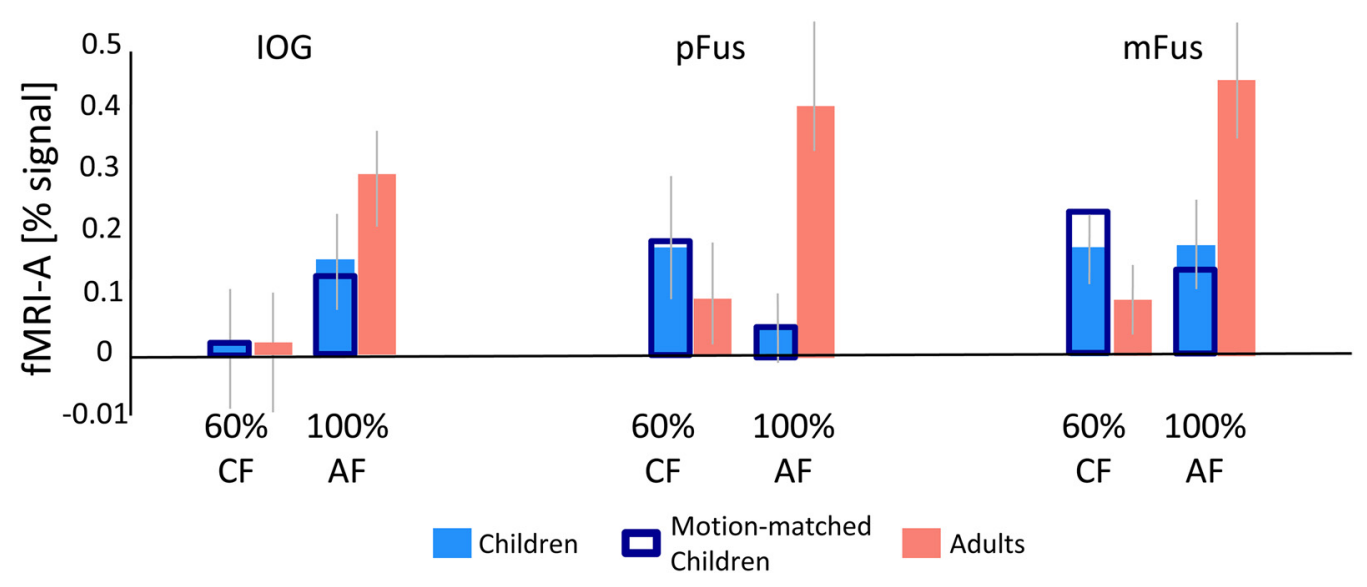

Figure 6. FMRI-A in face-selective regions shows an own-age bias. fMRI-A for IDI-matched faces of $60 \%$ morphs of child faces (CF) and 100\% adult faces (AF). Data are averaged across participants of each age group in each face-selective region. fMRI-A was measured as the signal difference between the condition of interest and blocks of identical child and adult faces, respectively. Orange, Mean fMRI-A in adults' face-selective regions averaged across 12 subjects in IOG, 12 subjects in pFus, and 12 subjects in mFus. Light blue, Mean fMRI-A in children's face-selective regions averaged across 17 subjects in IOG, 17 subjects in pFus, and 18 subjects in mFus. Dark Blue, Mean fMRI-A in the subset of 12 children motion-matched to adults. Error bars, SEM fMRI-A across participants of an age group.

and neural sensitivity to faces in children. Thus, in a conservative analysis, we removed seven children with highest motion under two voxels, matching age groups for motion (no significant difference in motion among matched groups: $F_{(1,45)}=3.41, p>$ $0.05 ; N_{\text {child }}=12, N_{\text {adult }}=12$ ) and repeated the analyses.

Analysis of responses of face-selective regions by morph level in this subset of motion-matched subjects replicate the results of the prior analyses. We found significantly larger slopes (main effect of age: $F_{(1,132)}=5.44, p<0.05$; Fig. $4 a$, dark blue bars) and higher baseline responses to faces (main effect of age: $F_{(1,132)}=$ $42.83, p<0.001$; Fig. $4 b$, dark blue bars) in adults' face-selective regions than in children's face-selective regions. Interestingly, in this subset of motion-matched subjects, slopes of neural sensitivity for own-age faces were significantly larger than slopes of neural sensitivity for other-age faces (age of subject by age of face interaction: $\left.F_{(1,132)}=4.37, p<0.05\right)$. Thus, this conservative analysis validated that the observed age-group differences in neural sensitivity and response amplitudes to faces in face-selective regions are not driven by group differences in motion during the scan.

Overall, we observed the following: (1) the responses of faceselective regions in both children and adults are sensitive to dissimilarity among face identity: responses in face-selective regions increase with increasing dissimilarity among faces; (2) baseline responses in face-selective regions to faces are larger in adults than in children regardless of the age of the face; and (3) neural sensitivity to face identity is higher in adults than in children, and in the motion-matched subjects, this neural sensitivity is larger for own-age faces than for other-age faces.

\section{Are differences between age groups due to low-level differences between face types?}

Results of the face-morph study show that in adults, responses to repetition of a single adult or child face produced the same amplitude of response in face-selective regions. A similar result was found in children. However, in adults, responses to $100 \%$ adult faces were higher than responses to $100 \%$ child faces (Fig. 3). We tested whether this was an outcome of differences in image dissimilarity among face stimuli types. Thus, we measured the IDI (see Materials and Methods) for all pairs of faces within each morph level and compared IDI across face types. Then we mea- sured the magnitude of fMRI-A (response in a condition of interest minus the response to identical faces) for IDI-matched child and adult faces.

Contrary to our prediction, the IDI of $100 \%$ adult faces was lower than the IDI of $100 \%$ different child faces $\left(t_{(10)}=2.99, p<\right.$ $0.05)$. In fact, the IDI of $100 \%$ different adult faces was comparable to the IDI of the $60 \%$ morph level of child faces $\left(t_{(10)}=0.8\right.$, $p>0.05)$. Examination of neural responses revealed that fMRI-A in face-selective regions for IDI-matched faces $(60 \%$ child vs $100 \%$ adults) was significantly larger in adults than in children (Fig. 6; main effect of age: all subjects, $F_{(1,164)}=5.07, p=0.025$; motion-matched subjects, $F_{(1,132)}=4.86, p=0.02$, three-way ANOVA with factors age of subject, age of face, and ROI). Additionally, fMRI-A for IDI-matched faces was also significantly larger for own-age faces than for other-age faces (age of subject by age of face interaction: all subjects, $F_{(1,164)}=10.89, p<0.01$; motion-matched subjects, $\left.F_{(1,132)}=17.11, p=0.001\right)$. We observed a similar pattern of results when comparing fMRI-A across age groups for $100 \%$ adult and $100 \%$ child faces (data not shown). Neural adaptation for $100 \%$ faces in face-selective regions was significantly larger in adults than in children (main effect of age: all subjects, $F_{(1,164)}=7.5, p<0.01$; motion-matched subjects, $F_{(1,132)}=9.52, p<0.01$, three-way ANOVA with factors age of subject, age of face, and ROI) and fMRI-A was also significantly larger for own-age faces than for other-age faces (age of subject-by-age of face interaction: all subjects, $F_{(1,164)}=7.96, p<$ 0.01 ; motion-matched subjects, $\left.F_{(1,132)}=8.52, p<0.01\right)$. These analyses show that both the larger fMRI-A in face-selective regions of adults than of children, and the more prominent ownage bias in adults, are likely not driven by low-level differences between stimuli of different face types.

Are developmental changes specific to face-selective regions? To address this question, we localized in each subject objectselective region (LO: $N_{\text {child }}=14 / 14, N_{\text {adult }}=10 / 10 ; \mathrm{pFs}: N_{\text {child }}=$ $13 / 14, N_{\text {adult }}=9 / 10$; see Materials and Methods) and examined their development from childhood to adulthood. Different from face-selective regions, there were no significant differences across age groups in the volume of $\operatorname{LO}\left(F_{(1,35)}=1.23, p>0.05\right)$ or $\mathrm{pFs}$ $\left(F_{(1,38)}=0.17, p>0.05\right)$. We next extracted responses from object-selective regions during the face-morph experiment. In 


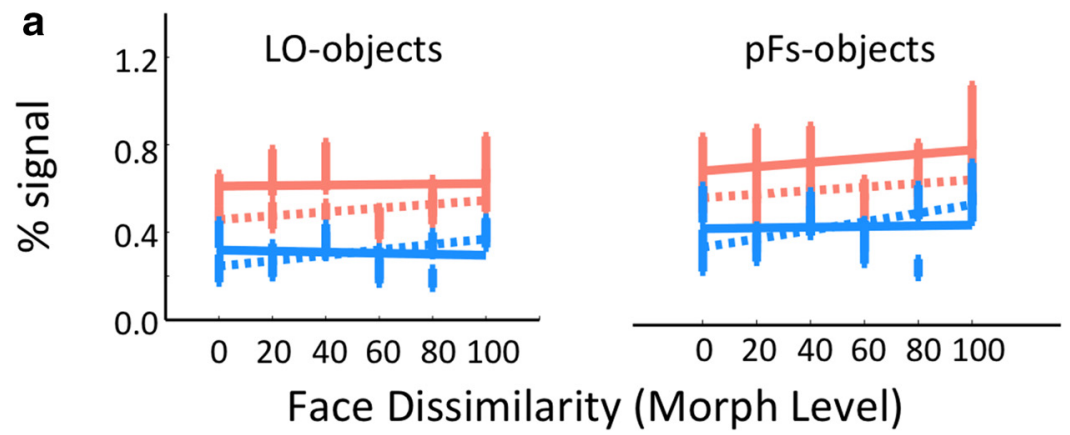




\section{a Dissimilarity ratings}

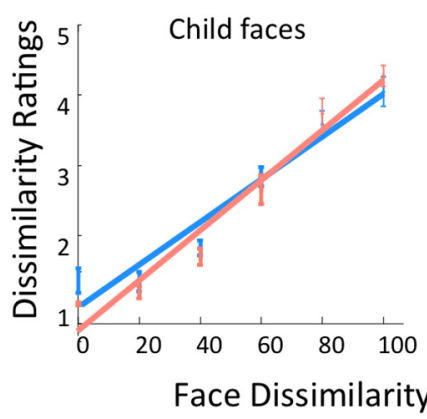

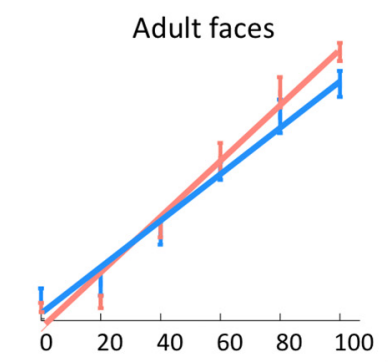

Children b Perceptual discriminability

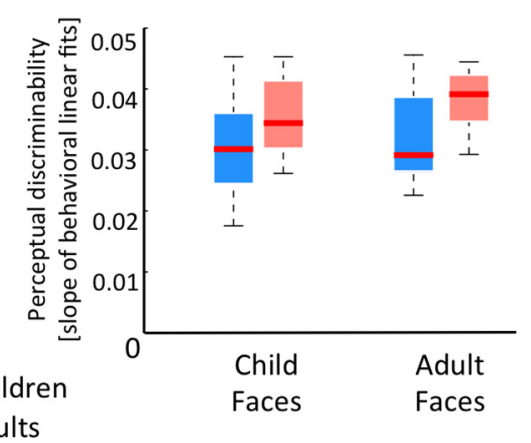

Figure 8. Perceptual dissimilarity ratings in children and adults. $\boldsymbol{a}$, Perceptual dissimilarity ratings across age groups: measurements and linear fits of the behavioral ratings of face dissimilarity as a function of morph level, separately for child faces (left) and adult faces (right). Blue, Children; orange, adults. Linear fits are the group average of individual subject fits. Error bars, SEM averaged across participants of an age group. $\boldsymbol{b}$, Perceptual discriminability across age groups: boxplots depicting median (red line), 25 and $75 \%$ percentiles (box edges), and range (dotted lines) of perceptual discriminability (slope of linear fit of dissimilarity ratings) in children (blue) and adults (orange). Adults have significantly higher perceptual discriminability than children across both face types $\left(F_{(1,42)}=8.95, p<0.01\right)$. In all plots, data are from 12 children and 11 adults.

$8 a$; main effect of morph level: $F_{(5,252)}=296.6, p<0.001$; threeway ANOVA on dissimilarity ratings with age of subject, age of face, and morph level as factors) and we found a significant age of subject-by-morph level interaction $\left(F_{(1,252)}=2.41, p<0.05\right)$. To assess perceptual discriminability, we measured in each subject the slope of the linear fit of the dissimilarity judgments versus morph level. We tested whether perceptual discriminability differed across children and adults. Results show significantly higher perceptual discriminability in adults than in children (main effect of age: $\left.F_{(1,42)}=8.95, p<0.01\right)$ even though some children reach the adult range of perceptual discriminability (Fig. $8 b$ ). Results replicate when perceptual data were fit with a Weibull function (main effect of age on slope of the Weibull function: $F_{(1,42)}=$ $4.38, p=0.0425)$. There was no significant main effect of age of face or a significant interaction between age of face and age of subject in either analysis.

We next tested whether perceptual dissimilarity judgments are related to neural sensitivity and/or baseline response levels in face-selective regions. Therefore, we measured the correlation between perceptual discriminability and neural sensitivity to face identity in face-selective regions (Fig. $4 a$ ) as well as the correlation between perceptual discriminability and baseline responses to faces (Fig. 4b). We repeated these analyses using data from object-selective regions to test whether responses to faces in object-selective regions also affect perceptual judgments of faces.

Results showed that subjects with higher neural sensitivity in face-selective regions had higher perceptual discriminability than those with lower neural sensitivity (Fig. 9). That is, we observed a positive correlation between perceptual discriminability of faces and neural sensitivity to faces in both pFus and mFus faces (child faces: pFus, $R=0.48, p=0.01$; mFus, $R=0.42, p=0.045$; Fig. $9 a$; adult faces: pFus, $R=0.54, p<0.01$; mFus, $R=0.58$, $p<$ 0.01 ; Fig. $9 b$ ). These positive correlations remained significant $(p s<0.05)$ when we regressed out subjects' ages. Correlations between perceptual sensitivity and neural discriminability also remained positive and significant in $\mathrm{pFus}$ faces for both face types (adult faces: $R=0.48, p=0.03$; child faces: $R=0.47, p=0.04$ ) and in mFus faces for adult faces (adult faces: $R=0.48, p=0.03$; child faces: $R=0.37 ; p=0.1$ ) when we removed three outlier child subjects who had negative neural slopes in these ROIs.

Examination of the relationship between perceptual discriminability to face identity versus baseline response level to faces in face-selective regions revealed no significant correlations in IOG faces and mFus faces $(-0.19<R<0.31 ; p s>0.05)$. We found a significant correlation in pFus faces (adult faces: $R=0.47, p=$ .02 ; child faces: $R=0.45, p=0.02$ ), but these correlations did not remain significant when we regressed out age. That is, subjects who had stronger responses in face-selective regions did not have better perceptual discriminability.

Different from face-selective regions, we did not observe a significant positive correlation between perceptual discriminability of faces and neural sensitivity to faces in objectselective regions $(0.2<R<0.39$; $p$ s $>0.05)$. Additionally, there was no significant correlation between perceptual discriminability of faces and response level to faces in objectselective regions $(-0.11<R<0.22$; $p$ s $>0.05)$.

Overall, these analyses reveal that specific development of neural sensitivity to face identity in face-selective regions is coupled with better perceptual discriminability for faces. However, development of the response level to faces is not coupled with better perceptual discriminability.

\section{Discussion}

Our study revealed three main findings: (1) neural sensitivity to face identity increases with age in face-selective but not objectselective regions, (2) the response amplitude to faces increases with age in both face-selective and object-selective regions, and (3) there is a correlation between neural sensitivity of faceselective regions to face identity and perceptual discriminability to face identity. These data suggest that both neural sensitivity and response amplitude to faces change from childhood to adulthood, but only neural sensitivity in face-selective regions correlates with perceptual discriminability. While domain-general factors of noise, motion, attention, motivation, and competence may affect BOLD signals in high-level visual cortex, these factors are unlikely to explain observed differences across children and adults for several reasons. First, we found no differences in signalto-noise ratios across age groups. Second, neural developmental effects remained stable and even strengthened when we motionmatched our participants and controlled stimuli for low-level and perceptual similarity. Third, children understood instructions and were competent at doing tasks. Fourth, our data show domain-specific development in three aspects: (1) within faceselective regions, responses in adults are higher than in children for face stimuli but not for other stimuli, (2) neural sensitivity to face identity develops in face-selective regions, but not object- 


\section{a Child Faces}

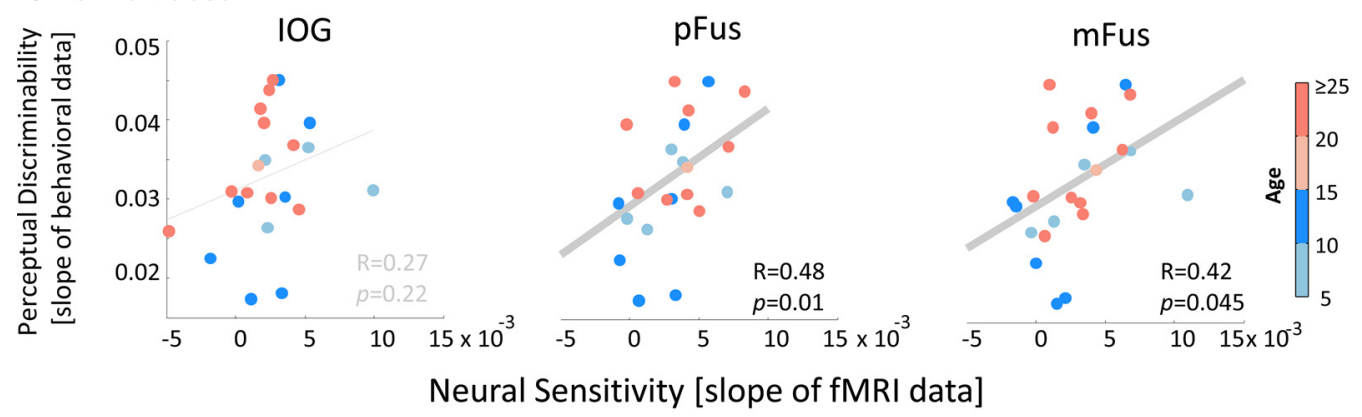

\section{b Adult Faces}
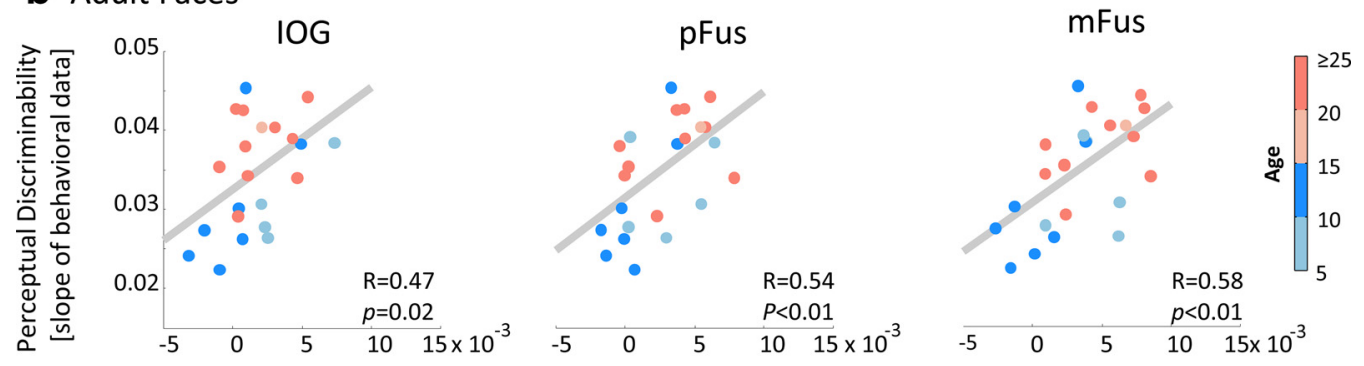

Neural Sensitivity [slope of fMRI data]

Figure 9. $\boldsymbol{a}, \boldsymbol{b}$, Correlation between perceptual discriminability versus neural sensitivity in IOG, pFus, and mFus faces in child $(\boldsymbol{a})$ and adult $(\boldsymbol{b})$ faces. Each point represents a participant, colored by age. There is a significant and positive correlation between perceptual and neural sensitivity to changes in face identity especially in pFus and $\mathrm{mFus}$ faces. Data are from 11 adults and 12 children, except for IOG faces, where we have data from 11 children because we were unable to localize the IOG faces in one child.

selective regions, and (3) perceptual discriminability is correlated with neural sensitivity in face-selective but not object-selective regions. Together, these results provide strong evidence that domain-general effects are not driving the reported developmental differences, as they would have produced similar effects across stimuli and brain regions.

\section{Development of neural sensitivity to changes in face identity is coupled with better face perception}

An important component of face perception is the ability to discriminate among faces and this ability develops with age (Mondloch et al., 2002; Weigelt et al., 2014). Face-selective regions also develop from childhood to adulthood (Aylward et al., 2005; Golarai et al., 2007, 2010; 2015; Scherf et al., 2007; Peelen et al., 2009; Cantlon et al., 2011). Our study is the first to examine how neural responses vary with parametric variations in face identity in children, revealing the following: (1) children's face-selective regions are sensitive to increasing dissimilarity among faces, as found in adults (Loffler et al., 2005; Jiang et al., 2006; Gilaie-Dotan et al., 2010; Davidenko et al., 2012); (2) neural sensitivity to face identity is present by age 5, and (3) children's face-selective regions exhibit fMRI-A, which is different from the lack of fMRI-A in children reported in prior studies (Scherf et al., 2011; Cohen Kadosh et al., 2013). Together these data suggest that responses in children's face-selective regions are quantitatively, not qualitatively, different from those of adults. We propose that neural adaptation to face exemplars might not be absent in children as suggested by Scherf et al. (2011), but instead increases in magnitude with age.

Notably, face discriminability was correlated with neural sensitivity in face-selective regions, but not baseline response to faces or neural sensitivity in object-selective regions. These findings suggest that developmental increases in neural sensitivity to faces in face-selective regions may underlie improved recognition abil- ities in adulthood. An open question is whether development of neural sensitivity occurs only for faces in face-selective regions or also for objects in object-selective regions, as behavioral studies suggest that perceptual discriminability of objects also develops (Weigelt et al., 2014).

While we find like prior studies (Mondloch et al., 2002; Weigelt et al., 2014) that perceptual discriminability of faces increases from childhood to adulthood, we acknowledge that children in our study (1) were 5 and older, (2) displayed qualitatively similar perceptual ratings as adults, and (3) showed significant face discriminability. Future research examining younger children and infants can elucidate the full trajectory of face discriminability development and whether larger developmental changes occur earlier in life (Crookes and McKone, 2009).

\section{Role of experience in shaping neural sensitivity for face identity}

Our results show higher neural sensitivity in adults' face-selective regions than in children's face-selective regions, and also a small but significant own-age bias in the following ways: (1) in children and adults who were motion-matched, neural sensitivity in faceselective regions was higher for own-age than for other-age faces, and (2) fMRI-A to identical faces relative to either $100 \%$ different child or adult faces or IDI-matched child and adult faces was higher for own-age than other-age faces.

Finding an own-age bias on top of a general age-related difference suggests that neural sensitivity to faces is partly shaped by experience. This finding is consistent with reports of experiencedriven development in humans (Golarai et al., 2015) and nonhuman primates (Srihasam et al., 2012, 2014) and an own-race bias in face-selective regions (Golby et al., 2001; de Heering et al., 2010; Natu et al., 2011). Our study does not elucidate what type of real life experiences contributes to the own-age bias because we did not quantify the amount of experience participants have with 
faces. However, we hypothesize that both recent exposure to own-age faces and cumulative exposure to faces across life may shape neural selectivity. For example, people encounter own-age faces more frequently than other-age faces (only one adult is a parent in our sample), own-age faces are socially salient (Hills and Lewis, 2011; Macchi Cassia, 2011), and adults have more cumulative experience with faces than children. Future studies examining neural and perceptual discriminability to faces in people who have extensive exposure to child faces, such as school teachers or pediatric nurses (de Heering and Rossion, 2008; Harrison and Hole, 2009), can elucidate the role of experience in shaping neural responses and perceptual abilities.

\section{What may be the neural mechanisms underlying the observed development in face-selective regions?}

We propose that increases in neural sensitivity to faces in faceselective regions may be associated with sharpening of neural tuning to face exemplars. This account suggests that children have broad tuning to face exemplars and with age and experience this neural tuning sharpens. Consequently, adults' face-selective regions show release from fMRI-A for smaller variations in face identity compared with children, leading to higher neural sensitivity in adults' face-selective regions compared with children. Although, we cannot directly measure neural tuning with fMRI, this hypothesis is consistent with empirical evidence in nonhuman primates showing that experience with objects narrows the tuning of neurons in the macaque inferotemporal (IT) cortex (Freedman et al., 2006).

Our observation of higher responses to faces in adults' faceselective regions than in children's face-selective regions across all morph levels may also be an outcome of narrower tuning to face exemplars in adults than in children. Specifically, broad neural tuning to faces in children would generate larger fMRI-A in children than in adults across morph levels, and consequently lower signals. Nevertheless, fMRI-A is not the only mechanism that may account for the lower responses to faces in children. Other neural mechanisms, such as age-related increases in the number of neurons that respond to faces and/or age-related increases in neural firing rates, may produce more elevated fMRI responses in adults than in children. Evidence for the former is supported by electrophysiological data in nonhuman primates showing that training increases the number of IT neurons responsive to trained stimuli (Kobatake et al., 1998). Future studies using technological advancements enabling recording from the same neuron over extended periods of time (McMahon et al., 2014) offer exciting possibilities to elucidate potential neural mechanisms of development in animal models.

\section{fMRI-A as a tool to test development of neural sensitivity to additional functional features}

As adaptation occurs at the neural level (Li et al., 1993; Sawamura et al., 2005, Kaliukhovich and Vogels, 2016), fMRI-A is a powerful tool that enables assessment of developmental changes to functional properties of neuronal populations within a region. Therefore, finding fMRI-A in children's face-selective regions opens new opportunities to investigate neural mechanisms of development of (1) additional aspects of face processing, (2) functional properties of other ventral-temporal regions, and (3) other high-level cognitive and sensory regions that exhibit fMRI-A (e.g., auditory cortex, ventrolateral prefrontal cortex).

In the domain of face processing, future fMRI-A developmental studies can test whether there are age-related developments in neural sensitivity to other aspects of face processing, such as con- figural processing (Yovel and Kanwisher, 2004; Schiltz and Rossion, 2006; Schiltz et al., 2006), expression (Winston et al., 2004), gaze (Calder et al., 2007), familiarity (Ewbank and Andrews, 2008; Avidan and Behrmann, 2009), or object transformations (Grill-Spector et al., 1999; Vuilleumier et al., 2003; Andrews and Ewbank, 2004). Sensitivity to these aspects can also be linked to development of perceptual abilities (Mondloch et al., 2003; Pellicano et al., 2006).

Importantly, using parametric fMRI-A, as we did here, brings developmental research to a new level as it can provide insights to tuning properties of neural populations. For example, parametric fMRI-A can test development of neural sensitivity in wordselective regions to letter case (Dehaene et al., 2001) or to bigram/ trigram frequency (Vinckier et al., 2007; Glezer et al., 2009, 2015) and its relationship to reading abilities.

In conclusion, our findings significantly advance understanding of the neural mechanisms underlying the development of face-selective regions in the ventral stream and their perceptual consequences and have important implications for future studies examining the development of neural representations in typical and atypical participants.

\section{References}

Andrews TJ, Ewbank MP (2004) Distinct representations for facial identity and changeable aspects of faces in the human temporal lobe. Neuroimage 23:905-913. CrossRef Medline

Avidan G, Behrmann M (2009) Functional MRI reveals compromised neural integrity of the face processing network in congenital prosopagnosia. Curr Biol 19:1146-1150. CrossRef Medline

Avidan G, Hasson U, Hendler T, Zohary E, Malach R (2002) Analysis of the neuronal selectivity underlying low fMRI signals. Curr Biol 12:964-972. CrossRef Medline

Aylward EH, Park JE, Field KM, Parsons AC, Richards TL, Cramer SC, Meltzoff AN (2005) Brain activation during face perception: evidence of a developmental change. J Cogn Neurosci 17:308-319. CrossRef Medline

Calder AJ, Beaver JD, Winston JS, Dolan RJ, Jenkins R, Eger E, Henson RN (2007) Separate coding of different gaze directions in the superior temporal sulcus and inferior parietal lobule. Curr Biol 17:20-25. CrossRef Medline

Cantlon JF, Pinel P, Dehaene S, Pelphrey KA (2011) Cortical representations of symbols, objects, and faces are pruned back during early childhood. Cereb Cortex 21:191-199. CrossRef Medline

Carey S, Diamond R, Woods B (1980) Development of face recognition-a maturational component. Dev Psychol 16:257-269. CrossRef

Cohen Kadosh K, Johnson MH, Dick F, Cohen Kadosh R, Blakemore SJ (2013) Effects of age, task performance, and structural brain development on face processing. Cereb Cortex 23:1630-1642. CrossRef Medline

Crookes K, McKone E (2009) Early maturity of face recognition: no childhood development of holistic processing, novel face encoding, or facespace. Cognition 111:219-247. CrossRef Medline

Dalrymple KA, Gomez J, Duchaine B (2013) The Dartmouth Database of Children's Faces: acquisition and validation of a new face stimulus set. PLoS One 8:e79131. CrossRef Medline

Davidenko N, Remus DA, Grill-Spector K (2012) Face-likeness and image variability drive responses in human face-selective ventral regions. Hum Brain Mapp 33:2334-2349. CrossRef Medline

Dehaene S, Naccache L, Cohen L, Bihan DL, Mangin JF, Poline JB, Rivière D (2001) Cerebral mechanisms of word masking and unconscious repetition priming. Nat Neurosci 4:752-758. CrossRef Medline

de Heering A, Rossion B (2008) Prolonged visual experience in adulthood modulates holistic face perception. PLoS One 3:e2317. CrossRef Medline

de Heering A, de Liedekerke C, Deboni M, Rossion B (2010) The role of experience during childhood in shaping the other-race effect. Dev Sci 13:181-187. CrossRef Medline

de Heering A, Rossion B, Maurer D (2012) Developmental changes in face recognition during childhood: evidence from upright and inverted faces. Cogn Dev 27:17-27. CrossRef

Ewbank MP, Andrews TJ (2008) Differential sensitivity for viewpoint be- 
tween familiar and unfamiliar faces in human visual cortex. Neuroimage 40:1857-1870. CrossRef Medline

Feinberg DA, Moeller S, Smith SM, Auerbach E, Ramanna S, Gunther M, Glasser MF, Miller KL, Ugurbil K, Yacoub E (2010) Multiplexed echo planar imaging for sub-second whole brain FMRI and fast diffusion imaging. PLoS One 5:e15710. CrossRef Medline

Freedman DJ, Riesenhuber M, Poggio T, Miller EK (2006) Experiencedependent sharpening of visual shape selectivity in inferior temporal cortex. Cereb Cortex 16:1631-1644. Medline

Gauthier I, Tarr MJ, Moylan J, Skudlarski P, Gore JC, Anderson AW (2000) The fusiform "face area" is part of a network that processes faces at the individual level. J Cogn Neurosci 12:495-504. CrossRef Medline

Gilaie-Dotan S, Malach R (2007) Sub-exemplar shape tuning in human face-related areas. Cereb Cortex 17:325-338. Medline

Gilaie-Dotan S, Gelbard-Sagiv H, Malach R (2010) Perceptual shape sensitivity to upright and inverted faces is reflected in neuronal adaptation. Neuroimage 50:383-395. CrossRef Medline

Glezer LS, Jiang X, Riesenhuber M (2009) Evidence for highly selective neuronal tuning to whole words in the "visual word form area." Neuron 62:199-204. CrossRef Medline

Glezer LS, Kim J, Rule J, Jiang X, Riesenhuber M (2015) Adding words to the brain's visual dictionary: novel word learning selectively sharpens orthographic representations in the VWFA. J Neurosci 35:4965-4972. CrossRef Medline

Golarai G, Ghahremani DG, Whitfield-Gabrieli S, Reiss A, Eberhardt JL, Gabrieli JD, Grill-Spector K (2007) Differential development of highlevel visual cortex correlates with category-specific recognition memory. Nat Neurosci 10:512-522. Medline

Golarai G, Liberman A, Yoon JM, Grill-Spector K (2010) Differential development of the ventral visual cortex extends through adolescence. Front Hum Neurosci 3:80. CrossRef Medline

Golarai G, Liberman A, Grill-Spector K (2015) Experience shapes the development of neural substrates of face processing in human ventral temporal cortex. Cereb Cortex pii:bhv314. CrossRef Medline.

Golby AJ, Gabrieli JD, Chiao JY, Eberhardt JL (2001) Differential responses in the fusiform region to same-race and other-race faces. Nat Neurosci 4:845-850. CrossRef Medline

Grill-Spector K, Malach R (2001) fMR-adaptation: a tool for studying the functional properties of human cortical neurons. Acta Psychol (Amst) 107:293-321. CrossRef Medline

Grill-Spector K, Kushnir T, Hendler T, Edelman S, Itzchak Y, Malach R (1998) A sequence of object-processing stages revealed by fMRI in the human occipital lobe. Hum Brain Mapp 6:316-328. CrossRef Medline

Grill-Spector K, Kushnir T, Edelman S, Avidan G, Itzchak Y, Malach R (1999) Differential processing of objects under various viewing conditions in the human lateral occipital complex. Neuron 24:187-203. CrossRef Medline

Grill-Spector K, Knouf N, Kanwisher N (2004) The fusiform face area subserves face perception, not generic within-category identification. Nat Neurosci 7:555-562. CrossRef Medline

Grill-Spector K, Golarai G, Gabrieli J (2008) Developmental neuroimaging of the human ventral visual cortex. Trends Cogn Sci 12:152-162. CrossRef Medline

Harrison V, Hole GJ (2009) Evidence for a contact-based explanation of the own-age bias in face recognition. Psychon Bull Rev 16:264-269. CrossRef Medline

Hills PJ, Lewis MB (2011) The own-age face recognition bias in children and adults. Q J Exp Psychol (Hove) 64:17-23. CrossRef Medline

Jiang X, Rosen E, Zeffiro T, Vanmeter J, Blanz V, Riesenhuber M (2006) Evaluation of a shape-based model of human face discrimination using FMRI and behavioral techniques. Neuron 50:159-172. CrossRef Medline

Kaliukhovich DA, Vogels R (2016) Divisive normalization predicts adaptation-induced response changes in macaque inferior temporal cortex. J Neurosci 36:6116-6128. CrossRef Medline

Kanwisher N, McDermott J, Chun MM (1997) The fusiform face area: a module in human extrastriate cortex specialized for face perception. J Neurosci 17:4302-4311. Medline

Kobatake E, Wang G, Tanaka K (1998) Effects of shape-discrimination training on the selectivity of inferotemporal cells in adult monkeys. J Neurophysiol 80:324-330. Medline

Li L, Miller EK, Desimone R (1993) The representation of stimulus famil- iarity in anterior inferior temporal cortex. J Neurophysiol 69:1918-1929. Medline

Loffler G, Yourganov G, Wilkinson F, Wilson HR (2005) fMRI evidence for the neural representation of faces. Nat Neurosci 8:1386-1390. CrossRef Medline

Macchi Cassia V (2011) Age biases in face processing: the effects of experience across development. Br J Psychol 102:816-829. CrossRef Medline

Malach R, Reppas JB, Benson RR, Kwong KK, Jiang H, Kennedy WA, Ledden PJ, Brady TJ, Rosen BR, Tootell RB (1995) Object-related activity revealed by functional magnetic resonance imaging in human occipital cortex. Proc Natl Acad Sci U S A 92:8135-8139. CrossRef Medline

McCarthy G, Puce A, Gore JC, Allison T (1997) Face-specific processing in the human fusiform gyrus. J Cogn Neurosci 9:605-610. CrossRef Medline

McMahon DB, Jones AP, Bondar IV, Leopold DA (2014) Face-selective neurons maintain consistent visual responses across months. Proc Natl Acad Sci U S A 111:8251-8256. CrossRef Medline

Mezer A, Yeatman JD, Stikov N, Kay KN, Cho NJ, Dougherty RF, Perry ML, Parvizi J, Hua le H, Butts-Pauly K, Wandell BA (2013) Quantifying the local tissue volume and composition in individual brains with magnetic resonance imaging. Nat Med 19:1667-1672. CrossRef Medline

Mondloch CJ, Le Grand R, Maurer D (2002) Configural face processing develops more slowly than featural face processing. Perception 31:553566. CrossRef Medline

Mondloch CJ, Geldart S, Maurer D, Le Grand R (2003) Developmental changes in face processing skills. J Exp Child Psychol 86:67-84. CrossRef Medline

Natu V, Raboy D, O'Toole AJ (2011) Neural correlates of own- and otherrace face perception: spatial and temporal response differences. Neuroimage 54:2547-2555. CrossRef Medline

Parvizi J, Jacques C, Foster BL, Withoft N, Rangarajan V, Weiner KS, GrillSpector K (2012) Electrical stimulation of human fusiform faceselective regions distorts face perception. J Neurosci 32:14915-14920. CrossRef Medline

Peelen MV, Glaser B, Vuilleumier P, Eliez S (2009) Differential development of selectivity for faces and bodies in the fusiform gyrus. Dev Sci 12:F16-F25. CrossRef Medline

Pellicano E, Rhodes G, Peters M (2006) Are preschoolers sensitive to configural information in faces? Dev Sci 9:270-277. CrossRef Medline

Reynolds JH, Heeger DJ (2009) The normalization model of attention. Neuron 61:168-185. CrossRef Medline

Rotshtein P, Henson RN, Treves A, Driver J, Dolan RJ (2005) Morphing Marilyn into Maggie dissociates physical and identity face representations in the brain. Nat Neurosci 8:107-113. CrossRef Medline

Sawamura H, Georgieva S, Vogels R, Vanduffel W, Orban GA (2005) Using functional magnetic resonance imaging to assess adaptation and size invariance of shape processing by humans and monkeys. J Neurosci 25 : 4294-4306. CrossRef Medline

Scherf KS, Behrmann M, Humphreys K, Luna B (2007) Visual categoryselectivity for faces, places and objects emerges along different developmental trajectories. Dev Sci 10:F15-F30. CrossRef Medline

Scherf KS, Luna B, Avidan G, Behrmann M (2011) "What" precedes "which": developmental neural tuning in face- and place-related cortex. Cereb Cortex 21:1963-1980. CrossRef Medline

Schiltz C, Rossion B (2006) Faces are represented holistically in the human occipito-temporal cortex. Neuroimage 32:1385-1394. CrossRef Medline

Schiltz C, Sorger B, Caldara R, Ahmed F, Mayer E, Goebel R, Rossion B (2006) Impaired face discrimination in acquired prosopagnosia is associated with abnormal response to individual faces in the right middle fusiform gyrus. Cereb Cortex 16:574-586. CrossRef Medline

Srihasam K, Mandeville JB, Morocz IA, Sullivan KJ, Livingstone MS (2012) Behavioral and anatomical consequences of early versus late symbol training in macaques. Neuron 73:608-619. CrossRef Medline

Srihasam K, Vincent JL, Livingstone MS (2014) Novel domain formation reveals proto-architecture in inferotemporal cortex. Nat Neurosci 17: 1776-1783. CrossRef Medline

Stigliani A, Weiner KS, Grill-Spector K (2015) Temporal processing capacity in high-level visual cortex is domain specific. J Neurosci 35:1241212424. CrossRef Medline

Vinckier F, Dehaene S, Jobert A, Dubus JP, Sigman M, Cohen L (2007) Hierarchical coding of letter strings in the ventral stream: dissecting the inner organization of the visual word-form system. Neuron 55:143-156. CrossRef Medline 
Vuilleumier P, Armony JL, Driver J, Dolan RJ (2003) Distinct spatial frequency sensitivities for processing faces and emotional expressions. Nat Neurosci 6:624-631. CrossRef Medline

Wang Z, Bovik AC, Sheikh HR, Simoncelli EP (2004) Image quality assessment: from error visibility to structural similarity. IEEE Trans Image Process 13:600-612. CrossRef Medline

Weigelt S, Koldewyn K, Dilks DD, Balas B, McKone E, Kanwisher N (2014) Domain-specific development of face memory but not face perception. Dev Sci 17:47-58. CrossRef Medline

Weiner KS, Grill-Spector K (2010) Sparsely-distributed organization of face and limb activations in human ventral temporal cortex. Neuroimage 52: 1559-1573. CrossRef Medline

Weiner KS, Grill-Spector K (2011) Not one extrastriate body area: using anatomical landmarks, hMT + , and visual field maps to parcellate limbselective activations in human lateral occipitotemporal cortex. Neuroimage 56:2183-2199. CrossRef Medline

Willenbockel V, Sadr J, Fiset D, Horne GO, Gosselin F, Tanaka JW (2010) Controlling low-level image properties: the SHINE toolbox. Behav Res Methods 42:671-684. CrossRef Medline

Winston JS, Henson RN, Fine-Goulden MR, Dolan RJ (2004) fMRI-adaptation reveals dissociable neural representations of identity and expression in face perception. J Neurophysiol 92:1830-1839. CrossRef Medline

Yovel G, Kanwisher N (2004) Face perception: domain specific, not process specific. Neuron 44:889-898. CrossRef Medline

Zychaluk K, Foster DH (2009) Model-free estimation of the psychometric function. Atten Percept Psychophys 71:1414-1425. CrossRef Medline 